\title{
Clinical applications of neurochemical and electrophysiological measurements for closed-loop neurostimulation
}

\author{
J. Blair Price, PhD, ${ }^{1}$ Aaron E. Rusheen, BSc, ${ }^{1,2}$ Abhijeet S. Barath, MBBS, ${ }^{1}$ \\ Juan M. Rojas Cabrera, BSc, ${ }^{1}$ Hojin Shin, PhD, ${ }^{1}$ Su-Youne Chang, PhD, ${ }^{1}$ \\ Christopher J. Kimble, MA, ${ }^{3}$ Kevin E. Bennet, PhD, MBA, ${ }^{1,3}$ Charles D. Blaha, PhD, ${ }^{1}$ \\ Kendall H. Lee, MD, PhD, ${ }^{1,4}$ and Yoonbae Oh, $\mathrm{PhD}^{1,4}$

\begin{abstract}
${ }^{1}$ Department of Neurologic Surgery, ${ }^{2}$ Medical Scientist Training Program, ${ }^{3}$ Division of Engineering, and ${ }^{4}$ Department of Biomedical Engineering, Mayo Clinic, Rochester, Minnesota
\end{abstract}

\begin{abstract}
The development of closed-loop deep brain stimulation (DBS) systems represents a significant opportunity for innovation in the clinical application of neurostimulation therapies. Despite the highly dynamic nature of neurological diseases, open-loop DBS applications are incapable of modifying parameters in real time to react to fluctuations in disease states. Thus, current practice for the designation of stimulation parameters, such as duration, amplitude, and pulse frequency, is an algorithmic process. Ideal stimulation parameters are highly individualized and must reflect both the specific disease presentation and the unique pathophysiology presented by the individual. Stimulation parameters currently require a lengthy trial-and-error process to achieve the maximal therapeutic effect and can only be modified during clinical visits. The major impediment to the development of automated, adaptive closed-loop systems involves the selection of highly specific disease-related biomarkers to provide feedback for the stimulation platform. This review explores the disease relevance of neurochemical and electrophysiological biomarkers for the development of closed-loop neurostimulation technologies. Electrophysiological biomarkers, such as local field potentials, have been used to monitor disease states. Real-time measurement of neurochemical substances may be similarly useful for disease characterization. Thus, the introduction of measurable neurochemical analytes has significantly expanded biomarker options for feedback-sensitive neuromodulation systems. The potential use of biomarker monitoring to advance neurostimulation approaches for treatment of Parkinson's disease, essential tremor, epilepsy, Tourette syndrome, obsessive-compulsive disorder, chronic pain, and depression is examined. Further, challenges and advances in the development of closed-loop neurostimulation technology are reviewed, as well as opportunities for next-generation closed-loop platforms.
\end{abstract}

https://thejns.org/doi/abs/10.3171/2020.4.FOCUS20167

KEYWORDS deep brain stimulation; closed loop; open loop; neurochemical measurement; electrophysiology; electrochemistry

$\mathrm{N}$ EUROSTIMULATION therapies such as deep brain stimulation (DBS) have evolved tremendously over the past decade. Since the first introduction of DBS, considerable progress has been made in refining the technique. This progress has prompted a multitude of descriptions regarding the primary mechanism of action of DBS, such as through inhibition of aberrant neural ac- tivity tied to cortical, limbic, or motor control, as well as modulation of neurochemical or electrophysiological activities. To examine these hypotheses, electrophysiological and electrochemical methods have been developed. These methods detect rapid or instantaneous physiological changes and thus can be used as biomarkers to develop closed-loop neurostimulation technologies. Electrophysi-

ABBREVIATIONS ACC = anterior cingulate cortex; $\mathrm{ALIC}=$ anterior limb of the internal capsule; $\mathrm{CM}-\mathrm{Pf}=$ centromedian-parafascicular complex; $\mathrm{DBS}=\mathrm{deep}$ brain stimulation; ET = essential tremor; FSCV = fast-scan cyclic voltammetry; GABA = gamma-aminobutyric acid; GPi = globus pallidus interna; LFP = local field potential; NAc = nucleus accumbens; $O C D$ = obsessive-compulsive disorder; PAG = periaqueductal gray; $P D=$ Parkinson's disease; PVG = periventricular gray matter; SANTE = Stimulation of the Anterior Nucleus of the Thalamus for Epilepsy; STN = subthalamic nucleus; TS = Tourette syndrome; VC = ventral capsule; VPL = ventral posterolateral nucleus; VPM = ventral posteromedial nucleus; VS = ventral striatum; vT = ventral thalamus; WINCS = Wireless Instantaneous Neurotransmitter Concentration Sensing.

SUBMITTED March 1, 2020. ACCEPTED April 16, 2020.

INCLUDE WHEN CITING DOI: 10.3171/2020.4.FOCUS20167. 


\section{A. Open-loop DBS}

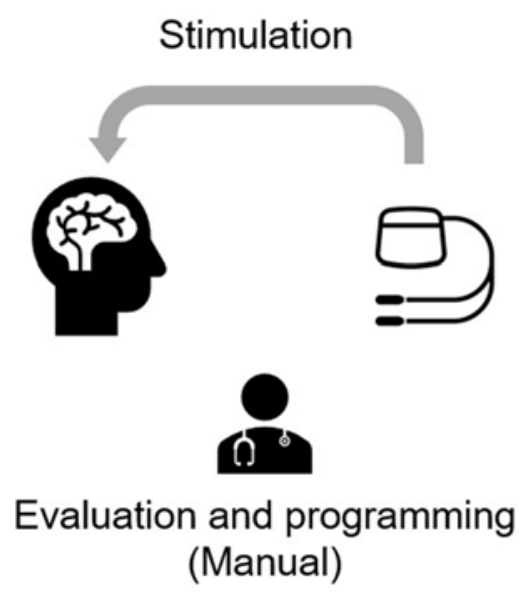

\section{B. Closed-loop DBS}

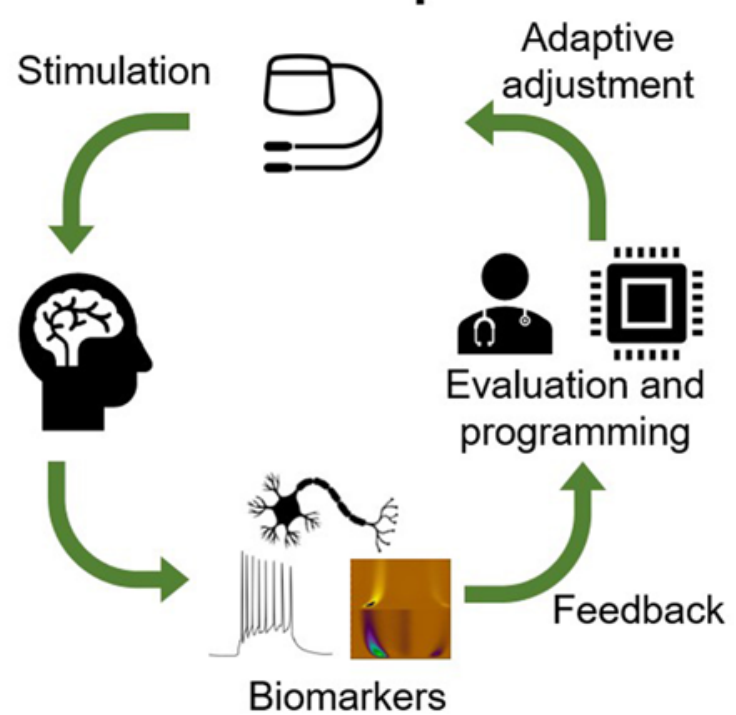

FIG. 1. Depiction of open- and closed-loop neurostimulation. A: Open loop. The patient is the primary source of feedback, and the clinician qualitatively evaluates the therapeutic efficacy of the stimulator. Providers algorithmically adjust the neurostimulation parameters. B: Closed loop. Quantitative feedback is provided by the biomarker sensor, and qualitative feedback is provided by the patient. Stimulation parameters are modified in real time by the neurostimulation platform with oversight by the clinician, thus providing feedback-sensitive, adaptive stimulation.

ological methods are used to measure electrical potentials to describe neuronal activities. Electrochemical measurements of electro-active species, such as catecholamines, are used to evaluate changes in extracellular neurotransmitter concentrations. While electrophysiological methods have received considerable clinical attention, electrochemical methods are still emerging for clinical applications due to the technological challenges of neurochemical recordings and, until recently, the inability to measure long-term tonic neurotransmitter levels. ${ }^{1}$

The pairing of neurostimulation and subsecond neurochemical measurements may usher in a wave of novel stimulation and recording techniques. Closed-loop neurostimulation methodologies, for which stimulation parameters are adapted by biomarker feedback, can streamline the individualization process of these treatments (Fig. 1). This, in turn, can modernize neurosurgical procedures for neurostimulation-based treatments. Here, we explore the recent advances toward the development of closed-loop neurostimulation systems using neurochemical feedback control. We review the clinical utility of neurochemicalsensitive neurostimulation therapies, novel advances in electrochemical recording techniques, and the development of closed-loop neurostimulation systems. Finally, we discuss opportunities and challenges in this field.

\section{Applications of Closed-Loop Neurostimulation in Neurosurgery}

Neurological disorders can be characterized by motor, behavioral, cognitive, affective, and perceptual traits that affect how individuals move, feel, think, and behave. Although most individuals with neurological disorders are successfully treated with medications and therapy, up to $30 \%$ of patients are unable to respond to standard therapeutic interventions. ${ }^{2}$ High-frequency electrical stimulation of subcortical brain structures with DBS is a highly successful alternative for treatment-resistant patients, ${ }^{3}$ such as for the treatment of Parkinson's disease (PD). Despite the clinical success of DBS, this method remains associated with limited efficacy and adverse treatment effects. These problems are partly due to poor manual programming of stimulation parameters. Presently, DBS systems are typically unidirectional open-loop devices, able only to provide nonadaptive stimulation according to predetermined parameters. These open-loop systems are uninformed by ongoing, time-sensitive input signals. Thus, the patient's therapeutic response to DBS is entirely dependent on clinician examination, requiring time-consuming iterative adjustments of stimulation parameters. ${ }^{1}$ Due to the lack of immediate feedback-sensitive adaptation, open-loop programming systems are significantly limited in their capacity to produce responsive, targeted stimulation. This technological shortcoming is holding back optimal care in DBS therapy as well as clinical expansion of this technology to complex neurological and psychiatric disorders.

Real-time, feedback-responsive modification of stimulation parameters therefore represents the next significant leap forward in the clinical application of DBS. Following surgery, closed-loop systems for DBS treatment are more effective, with fewer adverse side effects, such as dysarthria, than continuous stimulation. ${ }^{4}$ In a clinical study comparing closed- and open-loop DBS applications, it was found that closed-loop DBS-in response to beta power oscillations in the subthalamic nucleus (STN)-led 
to 50\% improvement in United Parkinson's Disease Rating Scale III (UPDRS-III) scores compared to $30 \%$ improvement with open-loop DBS. ${ }^{5,6}$ Further, total stimulation time by the closed-loop system was comparatively reduced, by $44 \%$. Battery drain is a significant concern with always-on approaches to DBS, as the constant power drain necessitates periodic replacement of the battery, which exposes the patient to repeated surgeries and infection risks. Feedback-sensitive approaches with closed-loop DBS preserve stimulator battery life, mitigating these risks. Thus, the identification of biomarkers suitable for informing stimulation parameters has emerged as a primary developmental goal for closed-loop technologies.

Neurochemical substances represent compelling biomarker targets for feedback-sensitive neurostimulation. In vivo electrochemical techniques have been used for decades to detect subsecond and submillimeter resolution changes in extracellular concentrations of neurochemicals, such as dopamine, serotonin, glutamate, and adenosine, in animal models. Further, abnormal neurochemical and electrophysiological activity has been implicated in a host of psychiatric and other neurological disorders, with relevant biomarkers for Parkinson's disease (PD), ${ }^{7}$ essential tremor (ET), ${ }^{8}$ epilepsy, ${ }^{9}$ Tourette syndrome (TS) ${ }_{1}^{10}$ obsessive-compulsive disorder (OCD), ${ }^{11}$ and depression. ${ }^{12}$

\section{Parkinson's Disease}

The therapeutic effects of DBS in PD are associated with the modulation of cortical and subcortical beta and gamma oscillations. ${ }^{713}$ Electrophysiological measurements in basal ganglia structures, as well as oscillatory activities of local field potentials (LFPs) in the dorsolateral motor region of the STN during functional neurosurgery, have provided critical insights into the pathophysiology of PD. Accordingly, closed-loop DBS systems, such as the Activa $\mathrm{PC}+\mathrm{S}$ (Medtronic) ${ }_{1}^{14}$ have focused on beta and gamma power as potential biomarkers for adaptive modulation of stimulation amplitude and frequency. These biomarkers guide feedback algorithms for closedloop DBS devices, enhancing the measurable outcomes of DBS. For example, a $27 \%$ improvement in motor outcome was achieved in patients with PD when STN DBS was applied only when beta power in electrophysiological activities reached a given threshold. ${ }^{5}$ Further, this closed-loop method halved the total activation duration compared to open-loop continuous stimulation.

To further develop closed-loop control of DBS for $\mathrm{PD}$, it is crucial to characterize the electrophysiological and neurochemical correlates of disease symptomology. Converging evidence suggests that DBS-induced excitation of efferent axons and fibers of passage results in network changes in dopamine neurotransmission. This process may also underlie parkinsonian motoric relief, ${ }^{15}$ determined via correction of abnormal oscillatory beta and gamma activity in basal ganglia-thalamocortical circuitry. Further, striatal phasic dopamine release has been shown in animal studies following short bursts of electrical stimulation of the STN and nucleus accumbens, common targets for DBS in PD and OCD patients, respectively. ${ }^{16,17}$ These studies suggest that striatal dopaminergic modulation may contribute to the therapeutic effect of DBS in PD.
Dopamine concentration monitoring in the striatum may therefore function as a potential feedback mechanism for neurochemistry-based closed-loop PD neurostimulation systems.

\section{Essential Tremor}

Unlike dopamine involvement in PD, the involvement of specific neurochemical systems in the pathophysiology of ET has not been established. Gamma-aminobutyric acid (GABA) receptor-mediated gap junction involvement $^{18}$ and serotonergic innervation ${ }^{19}$ in the inferior olivary nucleus (ION), as well as Purkinje cell loss in the cerebellar cortex, ${ }^{20}$ have been suggested as pathophysiological sources of tremor symptoms. However, these modulatory effects fail to fully account for the excitatory and inhibitory balance in the context of oscillatory neural activity between the thalamus and ION.

Due to the lack of understanding regarding pharmacological effects on ET, drug treatment options for ET and other types of tremor have not been developed to the point of targeting a specific neurochemical system. Thus, electrophysiological biomarkers have primarily been used for closed-loop DBS for tremor. However, in clinical trials, our group performed fast-scan cyclic voltammetry (FSCV) measurements in the ventral intermediate nucleus (VIM) of the thalamus of awake patients with ET in response to mechanical stimulation via the implanted DBS electrode. ${ }^{21,22} \mathrm{We}$ observed reduction in hand tremors, measured using a 3 -axis accelerometer, corresponding to a rapid, significant increase in adenosine oxidation currents. ${ }^{21,22}$ As a result, preclinical and clinical experiments have investigated adenosine as a potential target molecule for tremor therapy., ${ }^{9,22}$ Adenosine monitoring may therefore be used to optimize stimulation parameters to maximize therapeutic outcomes while reducing side effects. However, it is important to further describe the pathophysiology and neurochemical functions in tremor.

Neurotransmitter abnormalities in GABA, glutamate, dopamine, and noradrenaline transmission have also been implicated in the pathophysiology of ET.,23 Of these, the strongest evidence is for GABAergic dysfunction in the cerebellum. ${ }^{23}$ GABA, however, is nonelectroactive and thus cannot be detected via direct electrochemical techniques, such as amperometry or FSCV. Recently, a dual microbiosensor for measurement of GABA and glutamate has been described, with a limit of detection of $2 \pm 0.12$ $\mu \mathrm{M}$ for GABA. ${ }^{24}$ More research is required to further develop techniques with higher sensitivity for the detection of GABA at physiological concentrations. In addition, access to the cerebellum for GABA monitoring may prove challenging. Therefore, further investigation of additional candidate neurochemical biomarkers is warranted for the development of closed-loop DBS applications for ET.

\section{Epilepsy}

Ongoing research has led to continued characterization of additional viable biomarkers for epilepsy. One of the most pivotal clinical trials for DBS applications in epilepsy involved the Stimulation of the Anterior Nucleus of the Thalamus (ANT) for Epilepsy (SANTE) trial. ${ }^{25}$ The results were promising; after an initial blinded phase, pa- 
tients had a $40.4 \%$ median reduction in seizure frequency. At 5 years, Salanova et al. ${ }^{26}$ found the median seizure reduction had risen to $69 \%$ from baseline. However, many SANTE trial participants reported memory impairment. As a result, the fornix was postulated as an alternative target for DBS in patients with temporal lobe epilepsy. Subsequent studies ${ }^{27}$ employed bilateral, low-frequency stimulation of the fornix, leading to improvements in patients' hourly Mini-Mental State Examination (MMSE) scores. However, it was noted that factors such as antiepileptic medication confounded and the lack of sham controls complicated the interpretation of the results in these studies. Thus, further studies investigating possible targets for DBS in epilepsy patients are needed.

An additional obstacle in epilepsy treatment involves the identification of a reliable disease-relevant biomarker. Currently, specific types of electrophysiological activity, such as high-frequency oscillations, microseizures, and interictal spikes, are used as biomarkers for epilepsy. Monitoring these electrical activities in the brain makes it possible not only to distinguish epileptic and nonepileptic seizures, but also to identify epileptogenic brain tissue for resection or DBS targeting. However, there has been limited research investigating neurochemical opportunities for disease state monitoring. Recent studies using microdialysis have shown that during or following seizures, extracellular glutamate concentrations at the seizure site are increased, indicating a possible role in seizure-related brain structures. ${ }^{28}$ This finding presents a possible opportunity for feedback control following implantation of both stimulation and glutamate-recording electrodes into seizure sites. However, the precise mechanisms by which neurochemical substances contribute to seizure activity require further investigation.

\section{Tourette Syndrome}

TS is a neurodevelopmental disorder characterized by motor and vocal tics. While TS is primarily a childhood disease that improves throughout the developmental process, a subset of patients do not respond to medication and continue to display symptoms as adults. DBS has emerged as a treatment option for these severely affected patients. Two primary basal ganglia targets for DBS therapy have been identified: the globus pallidus interna (GPi) and the thalamic centromedian-parafascicular complex (CM-Pf). ${ }^{29,30}$

DBS treatment for TS has thus far been conducted in over 180 patients in whom there was a considerable reduction in tics. ${ }^{31}$ Our group recently conducted a retrospective examination of 10 patients with refractory TS and found that CM-Pf DBS improved motor tics by an average of $46 \%$ and phonic tics by an average of $52 \%$, as determined by using the Yale Global Tic Severity Scale (YGTSS). ${ }^{32,33}$ Studies have also demonstrated the efficacy of targeting the GPi. ${ }^{34}$ A recent double-blind crossover trial of $14 \mathrm{pa}-$ tients revealed a $15 \%$ reduction in tic severity as assessed by using the YGTSS. ${ }^{35}$ Further, a recent multisite retrospective study found significant improvements in YGTSS scores with both CM-Pf or GPi stimulation, with no differences in treatment outcomes between targets. ${ }^{31}$

Despite these successes, the therapeutic mechanism by which DBS acts is unknown. Research has focused primarily on DBS-enacted alterations of neurotransmission and neurochemical release. Depending on the stimulation target, it has been proposed that DBS affects circuits terminating in the striatum. This claim is supported by the discovery of increased activity in low-frequency and alpha bands in LFP recordings performed in the thalamus and GPi. ${ }^{36,37}$ The possibility of using LFP recordings from the CM-Pf during stimulation in a feed-forward system to adapt DBS parameters has also been explored. ${ }^{37}$ LFP recordings may therefore serve as suitable biomarkers for closed-loop stimulation platforms.

In addition to LFP recordings, circuits proposed for DBS modulation may regulate neurotransmitter concentrations. The primary neurotransmitters with evidence of pathologic concentration include dopamine, GABA, and glutamate. Of these, dopamine holds the most promise as a biomarker for neurostimulation feedback. The evidence for aberrant dopamine concentrations in TS includes a positive therapeutic effect of dopamine antagonists, increased dopamine transporter and vesicular monoamine transporter-2 binding with PET imaging, and increased dopamine release following amphetamine stimulation. ${ }^{38,39}$ Functional MRI studies of patients undergoing therapeutic stimulation have indicated hypoactivity in the striatum, a known structure with a high dopaminergic tone. ${ }^{40}$ Thus, striatal monitoring of extracellular dopamine during DBS has the potential to provide useful information. While the relationship between LFP, neurotransmitter levels, and DBS has yet to be uncovered, these variables may together represent multimodal opportunities for fine-tuning neurostimulation approaches for the treatment of TS.

\section{Chronic Pain}

Chronic pain, defined as pain extending beyond the typical timeframe of injury and healing, impacts $20.4 \%$ of adults in the United States. ${ }^{41}$ Chronic pain has been linked to opioid dependence, as well as the resultant opioid epidemic. ${ }^{42}$ Further, pain contributes to anxiety and depression, as well as reduced quality of life ${ }^{43}$ Chronic pain is not a well-delineated illness, and it features a multifaceted pathophysiology. A significant body of work has shown that central or peripheral neurological insults can lead to long-lasting pathologic changes in various brain regions involved in pain conduction. ${ }^{44}$ These regions can be divided into systems that process somatosensory, affective, and cognitive information. ${ }^{45}$ Due to the multidimensionality of pain, it can be difficult to adequately control chronic pain with behavioral therapy or pharmacotherapy. Thus, DBS has emerged as a potential treatment option in refractory patients.

Since the initial trials of DBS for pain, numerous studies for a myriad of pain indications have been performed. ${ }^{46}$ The most common DBS targets identified in these studies include the periaqueductal gray (PAG) and periventricular gray matter (PVG) region; the ventral thalamus (vT), which includes the ventral posterolateral nucleus (VPL) and ventral posteromedial nucleus (VPM); the anterior cingulate cortex (ACC); and the ventral striatum (VS) and anterior limb of the internal capsule (ALIC). The first multicenter randomized controlled trial of DBS for 
pain enrolled over 200 patients with a variety of chronic pain indications. ${ }^{47} \mathrm{DBS}$ was performed in either the vT or PAG. However, this trial failed to demonstrate significant improvement. Subsequent studies have been performed, with mixed results. ${ }^{46}$ In a successful study by Boccard et al., a mean pain reduction of $46 \%$ occurred after 1 year with PAG/PVG and/or VPL/VPM (vT) stimulation in 74 patients. ${ }^{48}$ Another study by Boccard et al. targeting the ACC found a $43.4 \%$ reduction after 1 year in 22 patients. ${ }^{49}$

However, suitable biomarkers for feedback control in DBS have yet to be identified. Potential chemical biomarkers may include GABA, dopamine, glutamate, or serotonin, as drugs affecting these neurotransmitter systems are currently used in pain treatment. ${ }^{50}$ Dopamine may offer the most potential for pain control monitoring due to its compatibility with neurochemical measurement methods; further, numerous imaging studies have shown aberrant striatal dopamine activity in pain sufferers..$^{51}$ Additionally, LFP recording has been proposed. In a recent report by Shirvalkar et al. ${ }^{45}$ these investigators proposed the recording of theta, alpha, and gamma oscillations in the S1 primary somatosensory cortex, the anterior cingulate cortex, and the orbitofrontal cortex as a way to predict chronic pain and adjust stimulation parameters. The extensive range of possible biomarkers, as well as the heterogeneous and unpredictable responses to DBS, are indicative that a closed-loop DBS system with fluid parameter optimization may be necessary to produce a reliable therapeutic response in cases of chronic pain.

\section{Obsessive-Compulsive Disorder}

The nucleus accumbens (NAc) is the most common target for DBS treatment of OCD. Other targets include the ventral capsule (VC) and ventral striatum (VS), the ALIC, the superolateral branch of the medial forebrain bundle, the amygdala, the STN, and the inferior thalamic peduncle. ${ }^{52,53}$ NAc DBS has also been performed to alleviate the obsessive-compulsive symptoms in autism spectrum disorders. ${ }^{53}$ NAc DBS modulates widespread cortical and subcortical networks in the cortico-striatothalmo-cortical circuit and, correspondingly, the levels of several neurotransmitters throughout the brain. NAc DBS has been shown to increase dopamine, serotonin, and noradrenaline in the prefrontal cortex,${ }^{54}$ increase dopamine in the striatum, ${ }^{55}$ and increase dopamine ${ }^{55}$ and GABA while reducing glutamate in the NAc. ${ }^{56}$

Monoamine abnormalities have been noted in animal models of OCD ${ }^{57}$ In clinical studies, selective serotonin reuptake inhibitors (SSRIs) and cognitive behavioral therapy were found to increase serotonin synthesis capability in widespread areas of the brain; further, increased serotonin synthesis in the raphe nucleus was associated with clinical improvement. ${ }^{58}$ SSRIs are the most effective medical treatment available for OCD, and dopamine antagonists have been used in some treatment-refractory cases. ${ }^{59,60}$ More recently, abnormalities of glutamate transmission have also been described in OCD patients. Subsequently, glutamate-modulating drugs have been trialed for treatment-refractory OCD. ${ }^{61}$

The evidence from neurochemical abnormalities in animal models, as well as current pharmacotherapies, treatment approaches, and studies of DBS mechanisms in OCD, suggest an overall picture of monoaminergic, glutamatergic, and GABAergic dysregulations in the pathophysiological effects of OCD. DBS of the NAc is associated with normalization of some of these abnormalities, leading to decreases in excitatory neurotransmission, increases in inhibitory neurotransmission, and complex modulation of the dopaminergic system. Dopamine, serotonin, and glutamate can be measured with high sensitivity with voltammetric techniques and biosensors, and thus are excellent candidate biomarkers for neurochemical-based closed-loop DBS systems. Recent advances from our group in developing electrochemical techniques for tonic dopamine and serotonin measurement further expand the armamentarium of research tools to study monoaminergic modulation with DBS. ${ }^{1,62}$ Further research aimed at correlating neurotransmitter concentrations with clinical improvement will provide the impetus needed to further develop closed-loop DBS systems.

\section{Depression}

Depression represents a challenging task for the development of closed-loop neurostimulation platforms. This is partly due to the ongoing debate regarding the optimal targets for stimulation, as well as the lack of reliable biomarkers with high disease specificity. Clinical trials have had mixed success; initial trials found therapeutic benefit following stimulation of areas such as the white matter tracts adjacent to the subgenual cingulate region (Brodmann area 25 [BA25] $)^{63}$ and the VC/VS. ${ }^{64}$ However, subsequent multicenter randomized controlled trials have failed to distinguish sham from stimulation in both BA25 $5^{65}$ and $\mathrm{VC} / \mathrm{VS},{ }^{66}$ dampening initial enthusiasm surrounding DBS for depression.

Though initially discouraging, subsequent research has suggested that the results of these trials may have been substantially impacted by variations in study design, probe placement, and stimulation parameters. For example, trials often compare sham and stimulation results following probe implantation, then subsequently provide open-label treatment. However, the inflammatory response to probe placement has been well documented to impart therapeutic effects regardless of stimulation, ${ }^{67}$ therefore presenting a significant confounder to this study design. Rather than directly comparing the therapeutic benefits between sham and stimulation following electrode implantation, an alternative design may provide all patients with open-label treatment before blinded discontinuation at a predefined time point. A randomized clinical trial featuring this crossover design found an initial $40 \%$ response rate in DBS of the ventral ALIC after 22 weeks. ${ }^{6}{ }^{8}$ Following blinded discontinuation, patients who continued stimulation scored significantly lower on the Hamilton Depression Rating Scale (HAM-D-17) than patients who discontinued stimulation.

In total, DBS holds significant promise as a therapeutic option for treatment-resistant depression. Fulfilling this promise, however, will require further definition of the therapeutic mechanism of DBS, as well as further refinement of the stimulation parameters. Optimizing closedloop systems will require careful selection of biomarkers 
to provide feedback for adaptive stimulation parameters. However, given the highly multimodal and individualized nature of depression, biomarker selection presents a significant challenge. The ideal biomarker will likely vary between presentations and subtypes of depression, necessitating individual pretesting of specific depression pathophysiology to define optimal stimulation parameters. Serotonin and dopamine may be viable options as biomarkers given their roles in depression neurochemistry and treatment. ${ }^{62}$ Alterations in dopamine and serotonin neurotransmission have been observed in the striatum and hippocampus, respectively, indicating possible regions for electrochemical monitoring. ${ }^{69,70}$

\section{Innovations and Challenges of Closed-Loop DBS Systems}

Though early indications of the effectiveness of closedloop DBS are highly promising, several challenges exist which must be addressed prior to widespread adoption of the technology. A major impediment to the development of closed-looped systems involves the selection and reliable measurement of ideal biomarkers for real-time evaluation of disease states. Ideal biomarker candidates should have high disease specificity and a high signal-to-noise ratio, should be unaffected by external conditions, and should be readily measurable over a long period across multiple disease states. ${ }^{7,71}$ Several biomarkers have been proposed for augmenting stimulation parameters, such as action potentials, LFPs, massed potentials, electrocorticographic activity, and neurotransmitter signaling. However, no single biomarker is sufficient to monitor progression of neurological disease symptomology or applications of DBS; further research is needed to isolate biomarkers and relate them to disease states and individualized pathophysiologies. Thus, a closed-loop system that can simultaneously monitor multiple biomarkers with established disease relevance may, therefore, achieve more granular monitoring of the disease state.

Another impediment to neurochemical monitoring by closed-loop systems is the degradation or fouling of sensors after their implantation in tissue. Over time, microelectrodes lose sensitivity for the targeted neurochemical analyte, necessitating removal and replacement of the microelectrode. Thus, longevity of the inserted probe is a key prerequisite for chronic monitoring of neurochemical substances in vivo. This issue has been addressed, in part, by developments in the electrode manufacturing process, which have led to enhanced detection capabilities for adsorption-controlled substances. For example, the deposition of polyethylenedioxythiophene (PEDOT) and polymers containing Nafion onto the surface of carbon fibers via electropolymerization remarkably increases sensitivity and selectivity for detection of electroactive molecules such as dopamine while mitigating electrode biofouling during in vivo recordings. ${ }^{72}$

The incorporation of novel materials has also paved the way for improved modifications to microsensors. One such example involves the use of boron-doped diamond microelectrodes in place of the conventional carbon-fiber microelectrodes. Studies conducted by our group have shown that diamond microelectrodes possess superior mechanical strength and increased longevity in vivo, and they may be a suitable option for chronic implantations and recordings. ${ }^{73}$ While the neurochemical sensitivity of these diamond microelectrodes was inferior to the sensitivity of carbon-fiber microelectrodes due to differences in the respective adsorption properties of the materials, it was shown that diamond microelectrodes maintain sensitivity to dopamine significantly longer than carbon-fiber microelectrodes. Carbon-fiber microelectrodes are quickly rendered unusable in vivo due to gradual surface degradation. Continuous improvements to microsensor fabrication techniques are necessary to overcome challenges in fouling and longevity inherent in established techniques.

\section{Future Directions and Innovations}

Our group is currently developing the next-generation Wireless Instantaneous Neurotransmitter Concentration Sensing (WINCS) system to incorporate the electrochemical and electrophysiological advances discussed above. This platform will enable the measurement of basal neurochemical concentrations while making concurrent measurements via FSCV, fixed-potential amperometry, and electrophysiology. The system is equipped with wireless Bluetooth and optical fiber links to communicate with base station software and provides multiple channels capable of sensing neurochemical concentration values and LFPs. This system is also capable of internally synchronizing stimulation and neurochemical sensing data while suppressing stimulation artifacts. Further development of this next-generation WINCS system will enable the system to interact with artificial neural network controllers, submitting voltammetric data to adapt stimulation parameters across dynamic shifts in disease states.

The combination of DBS with neurochemical sensing platforms such as WINCS ${ }^{74}$ can be used to fine-tune stimulation parameters for individualizing, modernizing, and possibly automating treatment methodologies for neurological and neuropsychiatric disorders. By evoking neural activity within disease-related neural structures, neurostimulation can facilitate the restoration of normal neural firing activity to enact clinical therapeutic effects.

\section{Conclusions}

Novel developments in electrochemistry work hand in hand with clinical practice to facilitate precise, individualized approaches for the designation of ideal neurostimulation parameters. However, limitations in our current stimulatory and electrochemical methods introduce hurdles that necessitate further research to overcome. There remains a critical need to investigate further the dynamic neurochemical states associated with neurological and neuropsychiatric diseases within a clinical context. Though preclinical work in the past decade has provided compelling evidence as to the efficacy of neurostimulation and its resultant influence on neurocircuitry, further clinical validations are needed to develop neurochemically based closed-loop stimulation platforms. If this is achieved, neurostimulation methodology can evolve from a primarily algorithmic method with restrictive biomarker 
options to an automated, feedback-sensitive, individualized method. This step, in turn, presents an opportunity to leap forward in the application of DBS for patient care of neurological and neuropsychiatric disorders.

\section{Acknowledgments}

This research was supported by NIH award nos. R01NS112176 and R01NS088260.

\section{References}

1. Oh Y, Heien ML, Park C, et al. Tracking tonic dopamine levels in vivo using multiple cyclic square wave voltammetry. Biosens Bioelectron. 2018;121:174-182.

2. Obeso JA, Olanow CW, Nutt JG. Levodopa motor complications in Parkinson's disease. Trends Neurosci. 2000;23(10 Suppl):S2-S7.

3. Almeida L, Deeb W, Spears C, et al. Current practice and the future of deep brain stimulation therapy in Parkinson's disease. Semin Neurol. 2017;37:205-214.

4. Little S, Beudel M, Zrinzo L, et al. Bilateral adaptive deep brain stimulation is effective in Parkinson's disease. J Neurol Neurosurg Psychiatry. 2016;87:717-721.

5. Little S, Pogosyan A, Neal S, et al. Adaptive deep brain stimulation in advanced Parkinson disease. Ann Neurol. 2013;74:449-457.

6. Kuo C-H, White-Dzuro GA, Ko AL. Approaches to closedloop deep brain stimulation for movement disorders. Neurosurg Focus. 2018;45(2):E2.

7. Bouthour W, Mégevand P, Donoghue J, et al. Biomarkers for closed-loop deep brain stimulation in Parkinson disease and beyond. Nat Rev Neurol. 2019;15:343-352.

8. Marin-Lahoz J, Gironell A. Linking essential tremor to the cerebellum: neurochemical evidence. Cerebellum. 2016;15:243-252.

9. Weltha L, Reemmer J, Boison D. The role of adenosine in epilepsy. Brain Res Bull. 2019;151:46-54.

10. Maia TV, Conceição VA. Dopaminergic disturbances in Tourette syndrome: an integrative account. Biol Psychiatry. 2018;84:332-344.

11. Bandelow B, Baldwin D, Abelli M, et al. Biological markers for anxiety disorders, OCD and PTSD: a consensus statement. Part II: Neurochemistry, neurophysiology and neurocognition. World J Biol Psychiatry. 2017;18:162-214.

12. Lee E-H, Han P-L. Reciprocal interactions across and within multiple levels of monoamine and cortico-limbic systems in stress-induced depression: a systematic review. Neurosci Biobehav Rev. 2019;101:13-31.

13. Swann NC, de Hemptinne C, Thompson MC, et al. Adaptive deep brain stimulation for Parkinson's disease using motor cortex sensing. J Neural Eng. 2018;15:046006.

14. Stanslaski S, Afshar P, Cong P, et al. Design and validation of a fully implantable, chronic, closed-loop neuromodulation device with concurrent sensing and stimulation. IEEE Trans Neural Syst Rehabil Eng. 2012;20:410-421.

15. McIntyre CC, Hahn PJ. Network perspectives on the mechanisms of deep brain stimulation. Neurobiol Dis. 2010;38:329337.

16. Figee M, de Koning P, Klaassen S, et al. Deep brain stimulation induces striatal dopamine release in obsessive-compulsive disorder. Biol Psychiatry. 2014;75:647-652.

17. Lee KH, Blaha CD, Harris BT, et al. Dopamine efflux in the rat striatum evoked by electrical stimulation of the subthalamic nucleus: potential mechanism of action in Parkinson's disease. Eur J Neurosci. 2006;23:1005-1014.

18. Ondo W. Essential tremor: what we can learn from current pharmacotherapy. Tremor Other Hyperkinet $\operatorname{Mov}(N Y)$. 2016;6:356.
19. Louis ED, Lenka A. The olivary hypothesis of essential tremor: time to lay this model to rest? Tremor Other Hyperkinet $\operatorname{Mov}(N Y)$. 2017;7:473.

20. Lin C-Y, Louis ED, Faust PL, et al. Abnormal climbing fibrePurkinje cell synaptic connections in the essential tremor cerebellum. Brain. 2014;137(12):3149-3159.

21. Chang S-Y, Kimble CJ, Kim I, et al. Development of the Mayo Investigational Neuromodulation Control System: toward a closed-loop electrochemical feedback system for deep brain stimulation. J Neurosurg. 2013;119:1556-1565.

22. Chang S-Y, Kim I, Marsh MP, et al. Wireless fast-scan cyclic voltammetry to monitor adenosine in patients with essential tremor during deep brain stimulation. Mayo Clin Proc. 2012;87:760-765.

23. Boecker H, Weindl A, Brooks DJ, et al. GABAergic dysfunction in essential tremor: an $11 \mathrm{C}$-flumazenil PET study. J Nucl Med. 2010;51(7):1030-1035.

24. Hossain I, Tan C, Doughty PT, et al. A novel microbiosensor microarray for continuous ex vivo monitoring of gammaaminobutyric acid in real-time. Front Neurosci. 2018;12:500.

25. Fisher R, Salanova V, Witt T, et al. Electrical stimulation of the anterior nucleus of thalamus for treatment of refractory epilepsy. Epilepsia. 2010;51:899-908.

26. Salanova V, Witt T, Worth R, et al. Long-term efficacy and safety of thalamic stimulation for drug-resistant partial epilepsy. Neurology. 2015;84:1017-1025.

27. Koubeissi MZ, Kahriman E, Syed TU, et al. Low-frequency electrical stimulation of a fiber tract in temporal lobe epilepsy. Ann Neurol. 2013;74:223-231.

28. Çavuş I, Romanyshyn JC, Kennard JT, et al. Elevated basal glutamate and unchanged glutamine and GABA in refractory epilepsy: microdialysis study of 79 patients at the Yale Epilepsy Surgery Program. Ann Neurol. 2016;80(1):35-45.

29. Xu W, Zhang C, Deeb W, et al. Deep brain stimulation for Tourette's syndrome. Transl Neurodegener. 2020;9:4.

30. Testini P, Min H-K, Bashir A, Lee KH. Deep brain stimulation for Tourette's syndrome: the case for targeting the thalamic centromedian-parafascicular complex. Front Neurol. 2016;7:193.

31. Martinez-Ramirez D, Jimenez-Shahed J, Leckman JF, et al. Efficacy and safety of deep brain stimulation in Tourette syndrome: The International Tourette Syndrome Deep Brain Stimulation Public Database and Registry. JAMA Neurol. 2018;75:353-359.

32. Testini P, Zhao CZ, Stead M, et al. Centromedian-parafascicular complex deep brain stimulation for Tourette syndrome: a retrospective study. Mayo Clin Proc. 2016;91:218-225.

33. Leckman JF, Riddle MA, Hardin MT, et al. The Yale Global Tic Severity Scale: initial testing of a clinician-rated scale of tic severity. J Am Acad Child Adolesc Psychiatry. 1989;28(4):566-573.

34. Martínez-Fernández R, Zrinzo L, Aviles-Olmos I, et al. Deep brain stimulation for Gilles de la Tourette syndrome: a case series targeting subregions of the globus pallidus internus. Mov Disord. 2011;26:1922-1930.

35. Kefalopoulou Z, Zrinzo L, Jahanshahi M, et al. Bilateral globus pallidus stimulation for severe Tourette's syndrome: a double-blind, randomised crossover trial. Lancet Neurol. 2015;14:595-605.

36. Jiménez-Sánchez L, Linge R, Campa L, et al. Behavioral, neurochemical and molecular changes after acute deep brain stimulation of the infralimbic prefrontal cortex. Neuropharmacology. 2016;108:91-102.

37. Marceglia S, Rosa M, Servello D, et al. Adaptive deep brain stimulation (aDBS) for Tourette syndrome. Brain Sci. 2017;8:E4.

38. Malison RT, McDougle CJ, van Dyck CH, et al. [123I]betaCIT SPECT imaging of striatal dopamine transporter binding in Tourette's disorder. Am J Psychiatry. 1995;152:1359-1361. 
39. Weisman H, Qureshi IA, Leckman JF, et al. Systematic review: pharmacological treatment of tic disorders-efficacy of antipsychotic and alpha-2 adrenergic agonist agents. Neurosci Biobehav Rev. 2013;37:1162-1171.

40. Jo HJ, McCairn KW, Gibson WS, et al. Global network modulation during thalamic stimulation for Tourette syndrome. Neuroimage Clin. 2018;18:502-509.

41. Dahlhamer J, Lucas J, Zelaya C, et al. Prevalence of chronic pain and high-impact chronic pain among adults-United States, 2016. MMWR Morb Mortal Wkly Rep. 2018;67:10011006.

42. Relieving pain in America: a blueprint for transforming prevention, care, education, and research. Mil Med. 2016;181(5):397-399.

43. Smith BH, Elliott AM, Chambers WA, et al. The impact of chronic pain in the community. Fam Pract. 2001;18:292-299.

44. Fornasari D. Pain mechanisms in patients with chronic pain. Clin Drug Investig. 2012;32(suppl 1):45-52.

45. Shirvalkar P, Veuthey TL, Dawes HE, Chang EF. Closedloop deep brain stimulation for refractory chronic pain. Front Comput Neurosci. 2018;12:18.

46. Frizon LA, Yamamoto EA, Nagel SJ, et al. Deep brain stimulation for pain in the modern era: a systematic review. Neurosurgery. 2020;86:191-202.

47. Coffey RJ. Deep brain stimulation for chronic pain: results of two multicenter trials and a structured review. Pain Med. 2001;2:183-192.

48. Boccard SGJ, Pereira EAC, Moir L, et al. Long-term outcomes of deep brain stimulation for neuropathic pain. Neurosurgery. 2013;72:221-231.

49. Boccard SGJ, Prangnell SJ, Pycroft L, et al. Long-term results of deep brain stimulation of the anterior cingulate cortex for neuropathic pain. World Neurosurg. 2017;106:625-637.

50. Lynch ME, Watson CPN. The pharmacotherapy of chronic pain: a review. Pain Res Manag. 2006;11:11-38.

51. Jääskeläinen SK, Rinne JO, Forssell H, et al. Role of the dopaminergic system in chronic pain-a fluorodopa-PET study. Pain. 2001;90:257-260.

52. Borders C, Hsu F, Sweidan AJ, et al. Deep brain stimulation for obsessive compulsive disorder: a review of results by anatomical target. Ment Illn. 2018;10:7900.

53. Karas PJ, Lee S, Jimenez-Shahed J, et al. Deep brain stimulation for obsessive compulsive disorder: evolution of surgical stimulation target parallels changing model of dysfunctional brain circuits. Front Neurosci. 2019;12:998.

54. van Dijk A, Klompmakers AA, Feenstra MGP, Denys D. Deep brain stimulation of the accumbens increases dopamine, serotonin, and noradrenaline in the prefrontal cortex. $J$ Neurochem. 2012;123:897-903.

55. Figee M, de Koning P, Klaassen S, et al. Deep brain stimulation induces striatal dopamine release in obsessive-compulsive disorder. Biol Psychiatry. 2014;75:647-652.

56. Yan N, Chen N, Zhu H, et al. High-frequency stimulation of nucleus accumbens changes in dopaminergic reward circuit. PLoS One. 2013;8:e79318.

57. Wood J, LaPalombara Z, Ahmari SE. Monoamine abnormalities in the SAPAP3 knockout model of obsessive-compulsive disorder-related behaviour. Philos Trans R Soc Lond B Biol Sci. 2018;373:373.

58. Lissemore JI, Sookman D, Gravel P, et al. Brain serotonin synthesis capacity in obsessive-compulsive disorder: effects of cognitive behavioral therapy and sertraline. Transl Psychiatry. 2018;8:82.

59. Soomro GM, Altman D, Rajagopal S, Oakley-Browne M. Selective serotonin re-uptake inhibitors (SSRIs) versus placebo for obsessive compulsive disorder (OCD). Cochrane Database Syst Rev. 2008;(1):CD001765.
60. Koo MS, Kim EJ, Roh D, Kim CH. Role of dopamine in the pathophysiology and treatment of obsessive-compulsive disorder. Expert Rev Neurother. 2010;10:275-290.

61. Pittenger C, Bloch MH, Williams K. Glutamate abnormalities in obsessive compulsive disorder: neurobiology, pathophysiology, and treatment. Pharmacol Ther. 2011;132:314332.

62. Shin H, Oh Y, Park C, et al. Sensitive and selective measurement of serotonin in vivo using fast cyclic square-wave voltammetry. Anal Chem. 2020;92(1):774-781.

63. Mayberg HS, Lozano AM, Voon V, et al. Deep brain stimulation for treatment-resistant depression. Neuron. 2005;45:651660.

64. Malone DA Jr, Dougherty DD, Rezai AR, et al. Deep brain stimulation of the ventral capsule/ventral striatum for treatment-resistant depression. Biol Psychiatry. 2009;65:267-275.

65. Holtzheimer PE, Husain MM, Lisanby SH, et al. Subcallosal cingulate deep brain stimulation for treatment-resistant depression: a multisite, randomised, sham-controlled trial. Lancet Psychiatry. 2017;4:839-849.

66. Dougherty DD, Rezai AR, Carpenter LL, et al. A randomized sham-controlled trial of deep brain stimulation of the ventral capsule/ventral striatum for chronic treatment-resistant depression. Biol Psychiatry. 2015;78:240-248.

67. Perez-Caballero L, Pérez-Egea R, Romero-Grimaldi C, et al. Early responses to deep brain stimulation in depression are modulated by anti-inflammatory drugs. Mol Psychiatry. 2014;19:607-614.

68. Bergfeld IO, Mantione M, Hoogendoorn MLC, et al. Deep brain stimulation of the ventral anterior limb of the internal capsule for treatment-resistant depression: a randomized clinical trial. JAMA Psychiatry. 2016;73:456-464.

69. Tye KM, Mirzabekov JJ, Warden MR, et al. Dopamine neurons modulate neural encoding and expression of depressionrelated behaviour. Nature. 2013;493:537-541.

70. Volle J, Bregman T, Scott B, et al. Deep brain stimulation and fluoxetine exert different long-term changes in the serotonergic system. Neuropharmacology. 2018;135:63-72.

71. Parastarfeizabadi M, Kouzani AZ. Advances in closedloop deep brain stimulation devices. J Neuroeng Rehabil. 2017;14(1):79.

72. Vreeland RF, Atcherley CW, Russell WS, et al. Biocompatible PEDOT:Nafion composite electrode coatings for selective detection of neurotransmitters in vivo. Anal Chem. 2015;87(5):2600-2607.

73. Bennet KE, Tomshine JR, Min H-K, et al. A diamond-based electrode for detection of neurochemicals in the human brain. Front Hum Neurosci. 2016;10:102.

74. Lee KH, Lujan JL, Trevathan JK, et al. WINCS Harmoni: Closed-loop dynamic neurochemical control of therapeutic interventions. Sci Rep. 2017;7:46675.

\section{Disclosures}

The authors designed the WINCS system described in the Future Directions and Innovations section.

\section{Author Contributions}

Conception and design: Oh, Price, Rusheen, Lee. Drafting the article: all authors. Critically revising the article: all authors. Reviewed submitted version of manuscript: all authors. Approved the final version of the manuscript on behalf of all authors: Oh.

\section{Correspondence}

Yoonbae Oh: Mayo Clinic, Rochester, MN. oh.yoonbae@mayo. edu. 